\title{
Dynamic Allan Variance Analysis Method with Time-Variant Window Length Based on Fuzzy Control
}

\author{
Shanshan Gu, ${ }^{1,2}$ Jianye Liu, ${ }^{1,2}$ Qinghua Zeng, ${ }^{1,2}$ Shaojun Feng, ${ }^{3}$ and Pin $\mathrm{Lv}^{1,2}$ \\ ${ }^{1}$ Jiangsu Key Laboratory of Internet of Things and Control Technologies, Nanjing University of Aeronautics and Astronautics, \\ 29 Yudao Street, Nanjing 210016, China \\ ${ }^{2}$ Navigation Research Center, College of Automation, Nanjing University of Aeronautics and Astronautics, \\ 29 Yudao Street, Nanjing 210016, China \\ ${ }^{3}$ Department of Civil and Environmental Engineering, Centre for Transport Studies, Imperial College London, \\ South Kensington Campus, London SW7 2AZ, UK
}

Correspondence should be addressed to Qinghua Zeng; zengqh@nuaa.edu.cn

Received 9 March 2015; Accepted 19 May 2015

Academic Editor: Geoffrey A. Cranch

Copyright (C) 2015 Shanshan Gu et al. This is an open access article distributed under the Creative Commons Attribution License, which permits unrestricted use, distribution, and reproduction in any medium, provided the original work is properly cited.

\begin{abstract}
To solve the problem that dynamic Allan variance (DAVAR) with fixed length of window cannot meet the identification accuracy requirement of fiber optic gyro (FOG) signal over all time domains, a dynamic Allan variance analysis method with time-variant window length based on fuzzy control is proposed. According to the characteristic of FOG signal, a fuzzy controller with the inputs of the first and second derivatives of FOG signal is designed to estimate the window length of the DAVAR. Then the Allan variances of the signals during the time-variant window are simulated to obtain the DAVAR of the FOG signal to describe the dynamic characteristic of the time-varying FOG signal. Additionally, a performance evaluation index of the algorithm based on radar chart is proposed. Experiment results show that, compared with different fixed window lengths DAVAR methods, the change of FOG signal with time can be identified effectively and the evaluation index of performance can be enhanced by $30 \%$ at least by the DAVAR method with time-variant window length based on fuzzy control.
\end{abstract}

\section{Introduction}

As the core of Inertial Navigation Systems (INS), fiber optic gyro (FOG) possesses the advantages of high reliability, impact resistance, high accuracy, and low power consumption $[1,2]$. However, the measurement accuracy of FOG is greatly deteriorated by the large random errors caused by the processing technology and working environment. It is of great necessity to analyze the error characteristics of FOG signal to identify the random errors and evaluate their performances [3]

The method of Allan variance is usually adopted to analyze and identify the random errors of FOG. As shown in [4], Allan variance is applied to evaluate the performance of a medium precision FOG. Allan variance is also employed to identify and model the five random errors of a practical FOG in [5]. Considering the nonstationarity of FOG signal caused by the influence of working environment such as temperature, humidity, and vibration, the results of Allan variance analysis cannot evaluate the varying error characteristics of FOG signal caused by these factors. To overcome the disadvantages of Allan variance, Galleani and Tavella in Politecnico di Torino of Italy developed the dynamic Allan variance (DAVAR) [6] which has been applied to be a representation of the time-varying stability of an atomic clock, and it can be used to monitor the clock behavior $[7,8]$.

The analysis accuracy of DAVAR is impacted greatly by the window length, where the shorter window length can reflect the characteristic of signal more faithfully and the longer window length can result in the higher confidence. So it is significant to select the reasonable window length for DAVAR analysis. Reference [6] discussed the analysis results of DAVAR to the nonlinear simulation signal under the different window length conditions. Besides, the influences 
of DAVAR to the dynamic error on different sway movements with different window lengths are analyzed in [9]. However, due to the nonstationarity of signal, it is impossible to obtain the high DAVAR analysis accuracy with the fixed window length and adaptive window size selection based on the signal characteristics is necessary. The above literatures just discussed DAVAR results of the signal under different window length conditions and did not give a method for adaptive window size selection.

The complex system, the characteristic of which is usually hard to be described, can be processed by fuzzy control. As a nonlinear intelligent control method with strong robustness and stability, fuzzy control has been used to learn and imitate human behavior and provide the objects which are difficult to be modeled with fuzzy inference and decision and is widely applied in navigation of unmanned aerial vehicles [10], mobile robots [11, 12], and so on.

Therefore, a DAVAR method with time-variant window length based on fuzzy control is proposed in this paper. A fuzzy controller decided by the first and second derivatives of FOG signal is implemented to estimate the optimum window length of DAVAR. Then the Allan variance with time-varying window length is realized to analyze the characteristics of real FOG signal. Finally, an index based on radar chart is designed to evaluate the performance of the proposed algorithm.

\section{DAVAR Theory}

As a time domain analysis tool, Allan variance is originally developed to study the frequency stability of oscillators and now is widely applied to identify the characteristics of the random errors that exist in the FOG signal. With the sampling frequency of $f$ (sampling time of $\tau_{0}$ ), the discrete-time Allan variance of collected FOG signal sample sequence of $\{x(t)\}$ is expressed as [6]

$$
\sigma_{\mathrm{AVAR}}^{2}(\tau)=\frac{1}{2}\left\langle\left(\bar{x}_{t_{k}+\tau}(\tau)-\bar{x}_{t_{k}}(\tau)\right)^{2}\right\rangle
$$

where the operator \langle\rangle denotes ensemble averaging, $\bar{x}_{t_{k}}(\tau)$ is the averaged measure of FOG signal at time $t_{k}$, and $\tau$ is observation interval.

Being the extension and improvement of Allan variance method, DAVAR is a representation of all the variances obtained at every time epoch and can be plotted in a single $3 \mathrm{D}$ graph. However, the Allan variance of random error is two dimensional and cannot reflect the nonstationarity of random errors of the inertial components output.

If the measurements are continuous with time, truncate the signal of $x(t)$ with the rectangular window length of $T$, and then build the increment process of $x_{T}\left(t, t^{\prime}, \tau\right)$ by convolving the truncated signal with the DAVAR window of $h_{\tau}\left(t^{\prime}\right)$ :

$$
x_{T}\left(t, t^{\prime}, \tau\right)=h_{\tau}\left(t^{\prime}\right) * x\left(t^{\prime}\right)
$$

where $*$ denotes convolution, $t^{\prime}$ is the central epoch of the DAVAR window, and $h_{\tau}\left(t^{\prime}\right)$ is the window of the Allan variance which is defined as follows:

$$
h_{\tau}\left(t^{\prime}\right)= \begin{cases}-\frac{1}{\tau}, & 0 \leq t^{\prime} \leq \tau, \\ \frac{1}{\tau}, & -\tau \leq t^{\prime} \leq 0,\end{cases}
$$

where

$$
t-\left(\frac{T}{2}-\tau\right) \leq t^{\prime} \leq t+\left(\frac{T}{2}-\tau\right) \quad 0<\tau \leq \tau_{\max } .
$$

Considering that the introduction of DAVAR window will lead to the frequency spectrum leakage and deteriorate the analysis accuracy and reliability, the maximum observation interval of $\tau_{\max }$ under the requirement of Allan variance estimation with window length of $T$ is [13]

$$
\tau_{\max }=\left\lfloor\frac{T}{3}\right\rfloor
$$

where \lfloor\rfloor indicates a operation of rounding down to the nearest integer.

Define DAVAR as the expectation of

$$
\sigma_{\text {DAVAR }}^{2}=\frac{1}{2} E\left[\left\langle x_{T}^{2}\left(t, t^{\prime}, \tau\right)\right\rangle\right],
$$

where the relationships among DAVAR, time of $t$, and correlation time of $\tau$ are established, and the $3 \mathrm{D}$ graph can be imaged.

\section{DAVAR with Time-Variant Window Length Based on Fuzzy Control}

DAVAR with single length of window function cannot meet the requirements of FOG signal identification accuracy over the whole time domains. Generally, short length of DAVAR window will follow fast time variation of the signal, but it will lead to large Allan variance error and cause the failure finally. On the other hand, long length of DAVAR window will make minor differences between DAVAR and Allan variance and fail to show the time variation of signal. Therefore, a method of adjustable DAVAR window lengths by employing fuzzy control is proposed to show the time variation of signal and guarantee the identification accuracy.

3.1. Design of Fuzzy Controller. Fuzzy controller is applied to infer the rules which are summarized by experience and experiment results. The proper fuzzy model and rules are the keys to obtain the ideal results [14]. The fuzzy values of inputs are separately assumed to be $E$ and $E C$, and the output is assumed to be $U$. To implement the fuzzy controller, the first and second derivatives of FOG signal are taken as the system inputs. Then the inputs are multiplied by quantization factors of $k_{e}$ and $k_{e c}$ separately to acquire the fuzzy values of $E$ and $E C$ which can be used to determine the control quantity of $U$ by lookup table of fuzzy control rules. Subsequently, the output of designed fuzzy controller is acquired with control quantity 


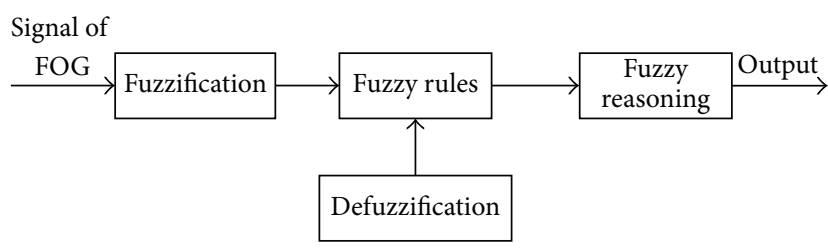

FIGURE 1: Principle diagram of fuzzy controller.

of $U$ divided by output factor of $k_{u}$ and the corresponding window length is determined. The whole designed fuzzy control, whose diagram is illustrated in Figure 1, consists of fuzzification, fuzzy rules, fuzzy reasoning, and defuzzification.

The fuzzy subsets of $E$ and $E C$ are of $\{\mathrm{NB}, \mathrm{NM}, \mathrm{NS}, \mathrm{ZO}$, $\mathrm{PS}, \mathrm{PM}, \mathrm{PB}\}$, which mean negative big (NB), negative medium (NM), negative small (NS), zero (ZO), positive small (PS), positive medium (PM), and positive big (PB). The fuzzy subset of the fuzzified output variable of $U$ is $\{\mathrm{ZO}, \mathrm{PS}, \mathrm{PM}, \mathrm{PB}\}$, which means zero (ZO), positive small (PS), positive medium (PM), and positive big (PB). In order to reduce the computational complexity, the membership functions of inputs and output are described by triangular membership functions as shown in Figure 2.

The basic universes of $E, E C$, and $U$ are separately assumed to be $\left[E_{\min }, E_{\max }\right],\left[E C_{\min }, E C_{\max }\right]$, and $\left[U_{\min }\right.$, $\left.U_{\max }\right]$, and the fuzzy universes are $[-3,3],[-3,3]$, and $[0,4]$; then

$$
\begin{aligned}
k_{e} & =\frac{6}{E_{\max }-E_{\min }}, \\
k_{e c} & =\frac{6}{E C_{\max }-E C_{\min }}, \\
k_{u} & =\frac{4}{U_{\max }-U_{\min }},
\end{aligned}
$$

where $k_{e}, k_{e c}$, and $k_{u}$ are the quantization factors of $E, E C$, and $U$.

According to the characteristic of real FOG signal and control experience, the fuzzy control rules are determined as shown in Table 1.

The fuzzy controller is modeled by Zadeh-Mamdani model, and the fuzzy rules can be described as "if $A$ and $B$ then $C$." The relationship $R$ can be described as

$$
R=A \times B \times C,
$$

where $A$ and $B$ are separately the fuzzy values of the first and second derivatives of FOG signal and $C$ is the fuzzy value of output.

The output of the fuzzy controller is obtained by $U$ which is determined by looking up the table of fuzzy control rules with $E$ and $E C$ and it can be written as

$$
y(n)=\frac{U}{k_{u}},
$$

where $y(n)$ is rounded to choose the appropriate length of DAVAR window conveniently. The rounding numbers of $y(n)$ are $1,2,3$, and 4 , which correspond to the DAVAR window
TABLE 1: Fuzzy control rules.

\begin{tabular}{lccccccc}
\hline$U$ & & & & $E$ & & & \\
& NB & NM & NS & ZO & PS & PM & PB \\
\hline$E C$ & & & & & & & \\
NB & PB & PB & PM & PM & PS & PS & ZO \\
NM & PB & PB & PM & PM & PS & ZO & PS \\
NS & PB & PB & PM & PS & ZO & PS & PM \\
ZO & PB & PM & PS & ZO & PS & PM & PB \\
PS & PM & PM & PS & ZO & PS & PM & PB \\
PM & PM & PS & ZO & PS & PM & PM & PB \\
PB & PS & ZO & PS & PM & PM & PB & PB \\
\hline
\end{tabular}

lengths of $T_{1}, T_{2}, T_{3}$, and $T_{4}$, respectively, and a set of the DAVAR windows lengths of $\left\{T_{t_{1}}, T_{t_{2}}, \ldots, T_{t_{3}}\right\}$ is acquired by fuzzy controller.

3.2. Calculation Steps of the Algorithm. The procedure of the DAVAR algorithm with time-variant window length based on fuzzy control can be summarized as follows:

(1) Acquire FOG signal, and calculate the set of the DAVAR windows lengths of $\left\{T_{t_{1}}, T_{t_{2}}, \ldots, T_{t_{3}}\right\}$ by fuzzy controller.

(2) Fix the analysis epoch at $t=t_{1}$.

(3) Truncate the FOG signal $x(t)$ with a window of length $T_{t_{1}}$ at $t_{1}$

(4) Evaluate the Allan variance $\sigma_{\mathrm{DAVAR}}^{2}\left(t_{1}, \tau\right)$.

(5) Choose another analysis epoch at $t=t_{2}$, truncate the signal by the rectangular window with width of $T_{t_{2}}$, and repeat from step (2). The new epoch $t_{2}$ can be chosen so that the corresponding truncated signal overlaps with the truncated signal related to the previous epoch $t_{1}$.

At the end of the above procedure, the collection of Allan variance $\sigma_{\mathrm{DAVAR}}^{2}\left(t_{1}, \tau\right), \sigma_{\mathrm{DAVAR}}^{2}\left(t_{2}, \tau\right), \ldots, \sigma_{\mathrm{DAVAR}}^{2}\left(t_{m}, \tau\right)$, related to the $m$ different epochs $t_{k}$ and to the different observation intervals $\tau$, gives a measure of the instantaneous stability of $x(t)$.

3.3. Algorithm Evaluation Indicators. In this paper, the radar chart, which is a two-dimensional chart for describing three or more variables using multiple axes from one point [15], is employed to evaluate the performance of the algorithm proposed. The size of radar chart indicates the performance states of object to be evaluated and it is helpful for diagnosis and control. Assuming all things are equal, small area of the radar chart represents good performance, and vice versa.

The radar chart of the proposed algorithm is composed by the performance parameters of time delay $\Delta t$ at the breakpoint, the average values of Allan variance coefficients of angle random walk $\bar{N}$, bias instability $\bar{B}$, rate random walk $\bar{K}$, rate ramp $\bar{R}$, and quantization noise $\bar{Q}$ when FOG 


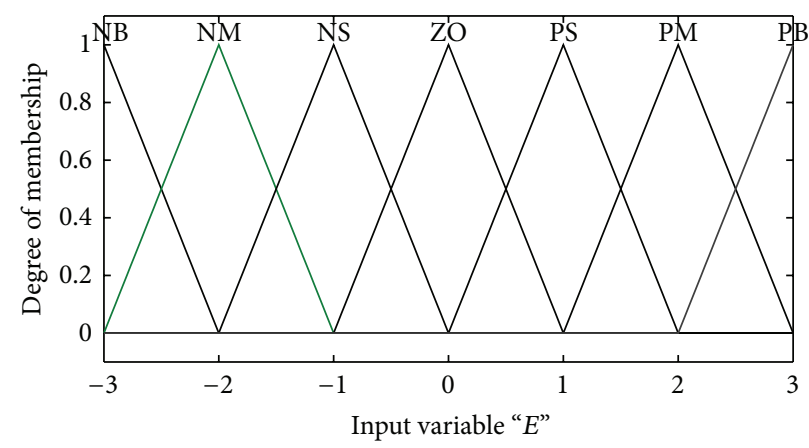

(a)

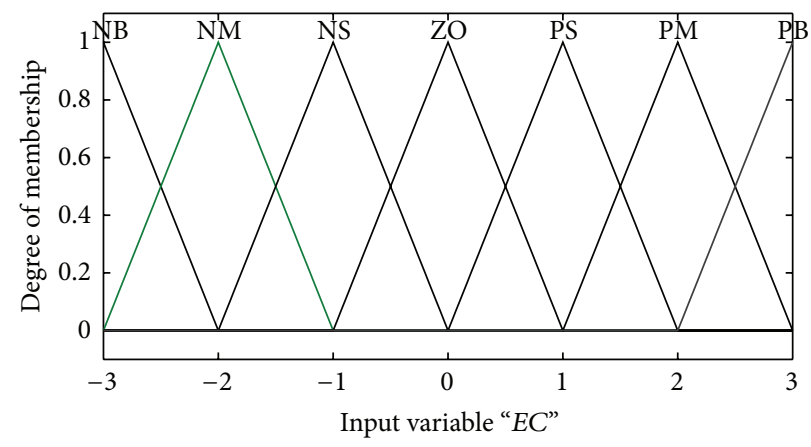

(b)

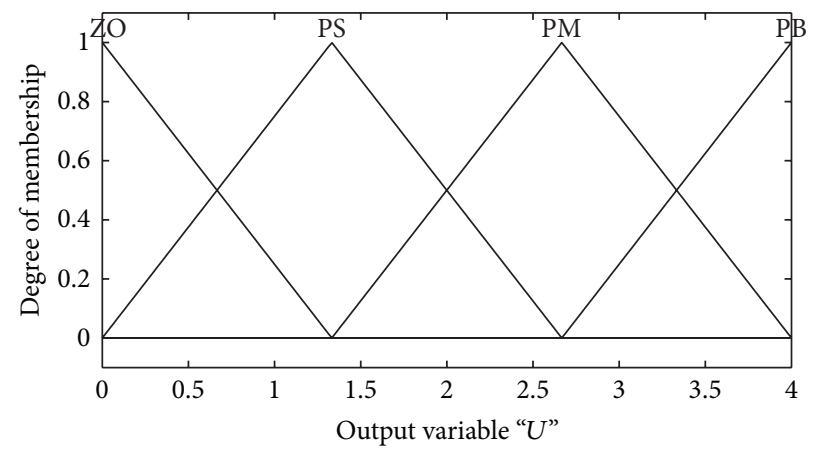

(c)

FIgURE 2: Membership functions of inputs and output: (a) input $E$, (b) input $E C$, and (c) output $U$.

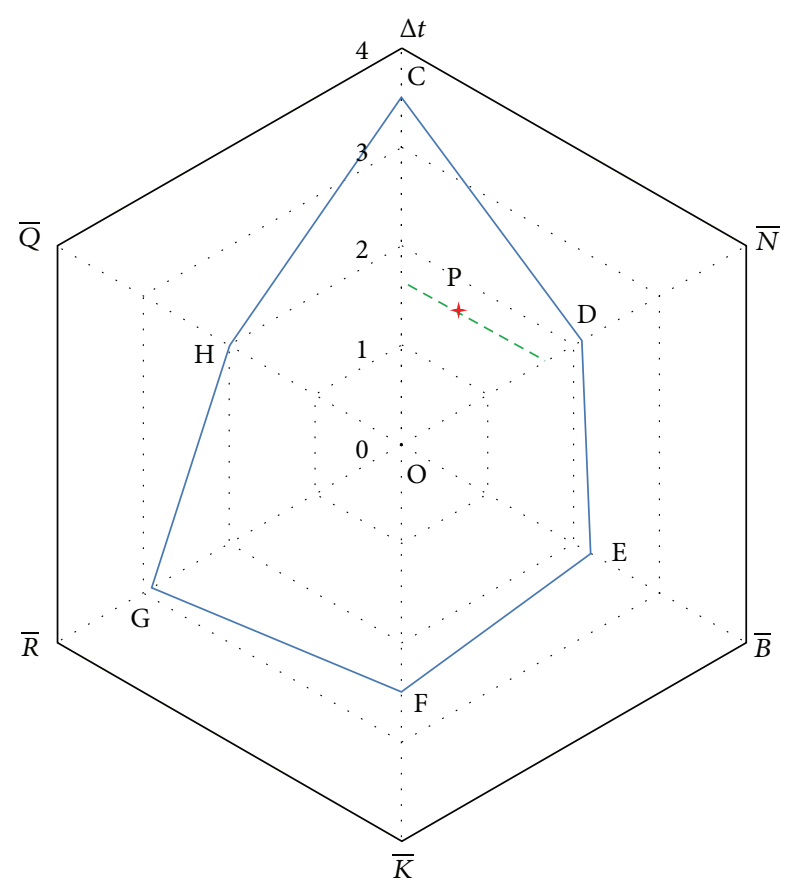

FIGURE 3: Illustration of triangle barycenter.

suffers vibration. Then the performance evaluation indicator is defined as

$$
\psi=\sum_{i=1}^{n} S_{i} \cdot w_{i}
$$

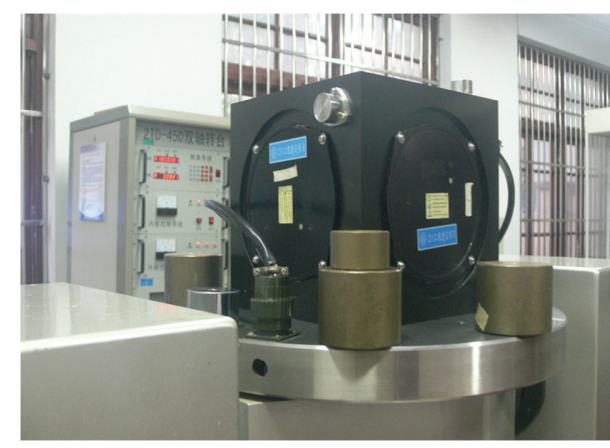

FIGURE 4: FOG on turntable.

where $n$ means the number of performance evaluation parameters, $S_{i}$ is the area of the triangle which is composed by six adjacent parameters of $\Delta t, \bar{N}, \bar{B}, \bar{K}, \bar{R}$, and $\bar{Q}$, and $w_{i}$ is the weight value of the triangle. As illustrated in Figure 3, the center of radar chart is assumed to be $O$, and the hexagon CDEFGH shows the performance of the algorithm to be evaluated, while the regular hexagons with the fine line represent the weight distributions. $S_{1}-S_{6}$ are the weights of triangle $\mathrm{OCD}, \mathrm{ODE}, \mathrm{OEF}, \mathrm{OFG}, \mathrm{OGH}$, and $\mathrm{OHC}$, respectively. The position of point $\mathrm{P}$, which is the barycenter of the triangle OCD, determines the weight of the triangle OCD.

The performance of DAVAR algorithm can be evaluated quantitatively by the performance evaluation indicator of which the smaller value means the better result in the same experiment. 


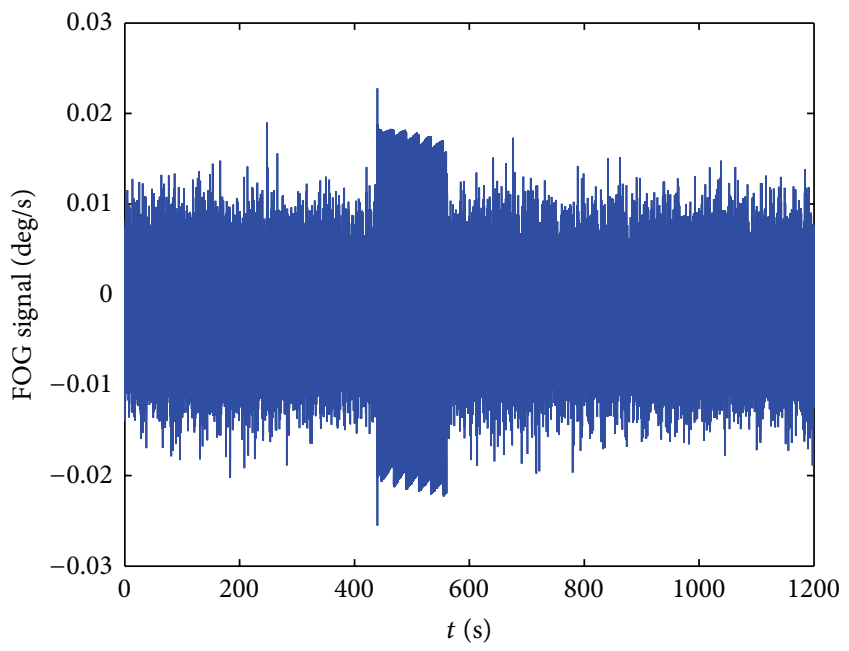

(a)

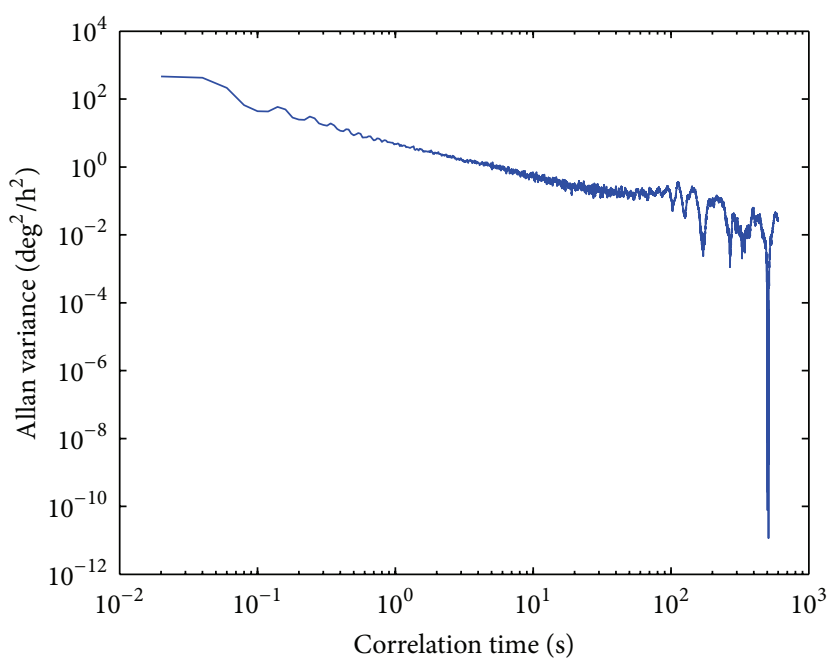

(b)

FIGURE 5: Signal and Allan variance of FOG: (a) FOG signal and (b) Allan variance.

Incorporating relationships between time and Allan variance coefficients, the proposed radar chart intuitionally shows the comprehensive characteristics of DAVAR results with different window lengths in a graphical way and evaluates the proposed DAVAR algorithm effectively by quantitative indicators.

\section{Results and Analysis}

To verify the proposed DAVAR algorithm, three groups of FOG signal on the turntable in the navigation laboratory of navigation research center (NRC) (as shown in Figure 4) under different experimental conditions are acquired with the sampling frequency of $50 \mathrm{~Hz}$ and the sampling duration of 1200 s. For the first group, the inner-frame is vibrated with certain amplitude and frequency during the period of $440 \mathrm{~s}-$ $560 \mathrm{~s}$. For the second and the third group, the outer-frame is vibrated during the periods of $440 \mathrm{~s}-560 \mathrm{~s}$ and $300 \mathrm{~s}-700 \mathrm{~s}$ separately.

Taking the $x$-axis signal in the first group as an example (shown as in Figure 5(a)), the vibration signal approximates to be a sinusoid. The ranges of inputs $E$ and $E C$ are $[-0.04,0.04]$ and $[-0.06,0.06]$ separately, and the range of output $U$ is $[0,4]$. As Figure 5 (a) shows, the noise of FOG signal is large during $440 \mathrm{~s}-560 \mathrm{~s}$, where the interference is more serious and the stability is poor. The result of traditional Allan variance is shown in Figure 5(b) and the nonstationary variation cannot be observed.

The different lengths of DAVAR window are adopted to analyze the FOG signal, respectively, and the corresponding analysis results are shown in Figure 6. With the short window length DAVAR algorithm (SDAVAR), there are obvious fluctuations of DAVAR curves. When the long window length DAVAR algorithm (LDAVAR) and timevarying lengths DAVAR algorithm (proposed algorithm) are employed, the fluctuations of proposed algorithm are less than SDAVAR but larger than LDAVAR.
TABLE 2: Performance evaluation indicators of radar chart.

\begin{tabular}{lccccccc}
\hline & $\Delta t$ & $\bar{N}$ & $\bar{B}$ & $\bar{K}$ & $\bar{R}$ & $\bar{Q}$ & $\psi$ \\
\hline SDAVAR & 1 & 1.25 & 1.51 & 2.21 & 3.79 & 1.22 & 14.19 \\
LDAVAR & 4 & 1.00 & 1.00 & 1.00 & 1.00 & 1.00 & 7.26 \\
Proposed algorithm & 2 & 1.10 & 1.20 & 1.46 & 1.91 & 1.09 & 5.37 \\
\hline
\end{tabular}

TABLE 3: Weights of triangles in radar chart.

\begin{tabular}{lcccccc}
\hline & $w_{1}$ & $w_{2}$ & $w_{3}$ & $w_{4}$ & $w_{5}$ & $w_{6}$ \\
\hline SDAVAR & 0.75 & 0.92 & 1.25 & 2.02 & 1.74 & 0.74 \\
LDAVAR & 1.76 & 0.67 & 0.67 & 0.67 & 0.67 & 1.76 \\
Proposed algorithm & 1.05 & 0.77 & 0.89 & 1.13 & 1.01 & 1.04 \\
\hline
\end{tabular}

Figure 7 shows the relationships among the Allan variance coefficients obtained by the three algorithms. It can be seen that the Allan variance coefficients are not constants. During the period of $0-440 \mathrm{~s}$, the blue solid line shows the result of SDAVAR, the dynamic characteristic of which is obvious. The green dotted line paints the result of LDAVAR window, which is the smoothest. The red imaginary line describes the result of the proposed algorithm, which is smoother than the blue one, but some dynamic characteristics are still retained. Nearby the time of $440 \mathrm{~s}$, the time delay of SDAVAR is $2 \mathrm{~s}$, which is the smallest; while the delay of proposed algorithm is $4 \mathrm{~s}$, the time delay of LDAVAR window is $8 \mathrm{~s}$. During the period of $440 \mathrm{~s}-560 \mathrm{~s}$, the Allan variance coefficients of SDAVAR are the largest, and those of proposed algorithm are in the middle.

To compare the performances of the three DAVAR algorithms of SDAVAR, LDAVAR, and proposed algorithm, the performance evaluation index is calculated by radar chart. The parameters of radar chart are time delay of $\Delta t$ at the breakpoint and the average values of Allan variance coefficients of $\bar{N}, \bar{B}, \bar{K}, \bar{R}$, and $\bar{Q}$. For convenience, the 


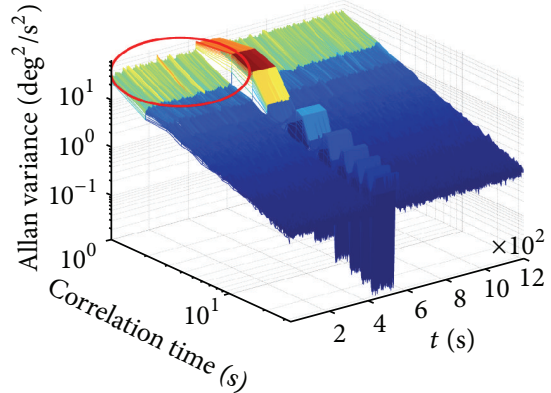

(a)

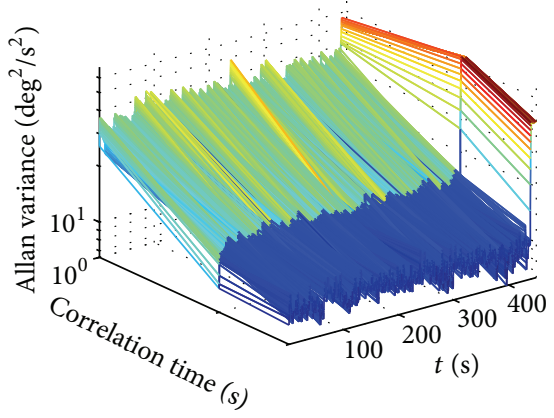

(d)

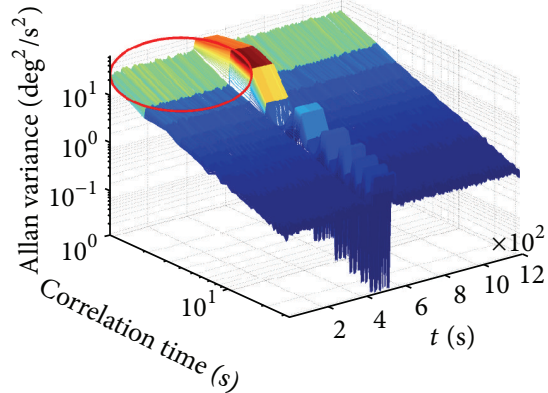

(b)

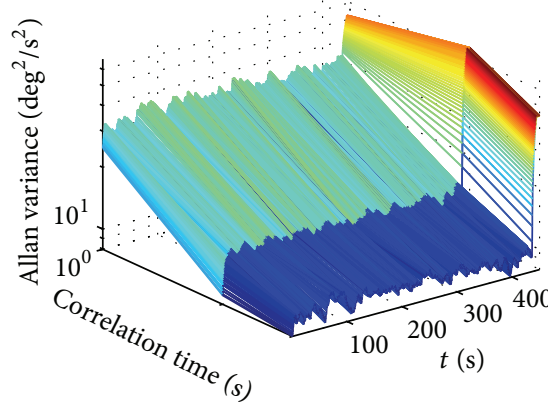

(e)

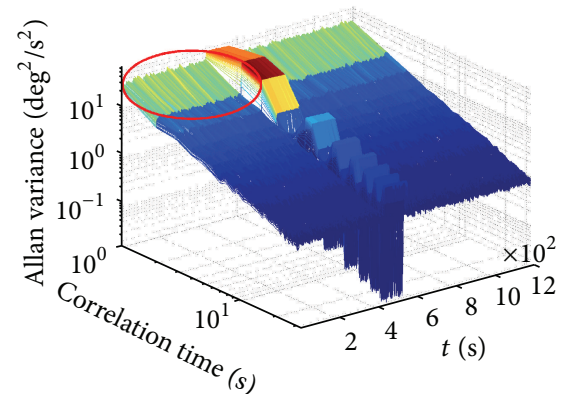

(c)

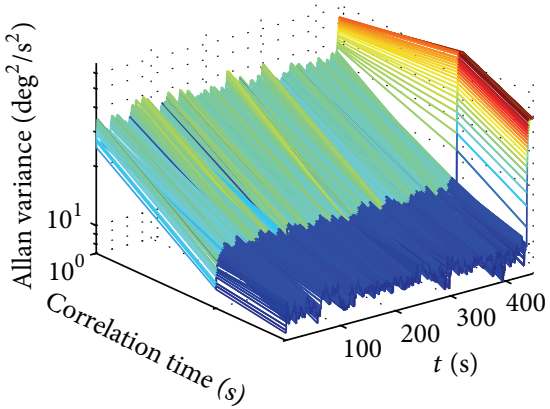

(f)

FIGURE 6: DAVAR of FOG: (a) SDAVAR, (b) LDAVAR, (c) proposed algorithm, (d) detail of SDAVAR, (e) detail of LDAVAR, and (f) detail of proposed algorithm.

TABLE 4: Performance evaluation indicators of radar chart.

\begin{tabular}{|c|c|c|c|c|c|c|c|}
\hline & $\Delta t$ & $\bar{N}$ & $\bar{B}$ & $\bar{K}$ & $\bar{R}$ & $\bar{Q}$ & $\psi$ \\
\hline \multicolumn{8}{|c|}{$y$-axis signal of the second group } \\
\hline SDAVAR & 1 & 1.25 & 1.51 & 2.20 & 3.78 & 1.22 & 14.03 \\
\hline LDAVAR & 4 & 1.00 & 1.00 & 1.00 & 1.00 & 1.00 & 7.26 \\
\hline Proposed algorithm & 2 & 1.10 & 1.20 & 1.46 & 1.91 & 1.09 & 5.36 \\
\hline \multicolumn{8}{|c|}{$z$-axis signal of the third group } \\
\hline SDAVAR & 1 & 1.26 & 1.51 & 2.22 & 3.80 & 1.22 & 14.33 \\
\hline LDAVAR & 4 & 1.00 & 1.00 & 1.00 & 1.00 & 1.00 & 7.26 \\
\hline Proposed algorithm & 2 & 1.10 & 1.20 & 1.46 & 1.92 & 1.09 & 5.39 \\
\hline
\end{tabular}

smallest values of every kind of parameters are treated as 1 , and the parameters are normalized as shown in Table 2. Then algorithm performance evaluation indicators are calculated as shown of $\psi$ in Table 2. Weights of triangles composed by $\Delta t, \bar{N}, \bar{B}, \bar{K}, \bar{R}$, and $\bar{Q}$ in turn are shown in Table 3. Figure 8 shows the radar chart of algorithm performance evaluation. It can be seen that the indicator of proposed algorithm is the smallest, which is improved by $67 \%$ compared with LDAVAR and $37 \%$ compared with SDAVAR. Obviously the proposed algorithm has better performances than the others.

Table 4 lists the radar chart of the $y$-axis signal in the second group and the $z$-axis signal in the third group. Figure 9 compares the performance evaluation indicators of the three signals, which show that the performance evaluation indicators of the three signals are approximate with the same length of DAVAR window, and the best performance evaluation indicators can be obtained by the proposed algorithm.
The parameters and performance evaluation indicators of other signals are similar to the present ones, so it is not necessary to enumerate all the discussions here.

\section{Conclusion}

In this paper, a DAVAR algorithm with time-variant window length determined by the designed fuzzy control is proposed, which incorporates the advantages of DAVAR algorithms with long and short window lengths. The given experiment results demonstrate that the precise variation characteristics analysis of FOG signal errors is implemented and the accuracy of the algorithm is ensured. Also an indicator based on the radar chart is also presented to evaluate the performance of the algorithm. Compared with LDAVAR and SDAVAR, the performance indicator of time-varying length is enhanced by $67 \%$ and $37 \%$ separately. The proposed 

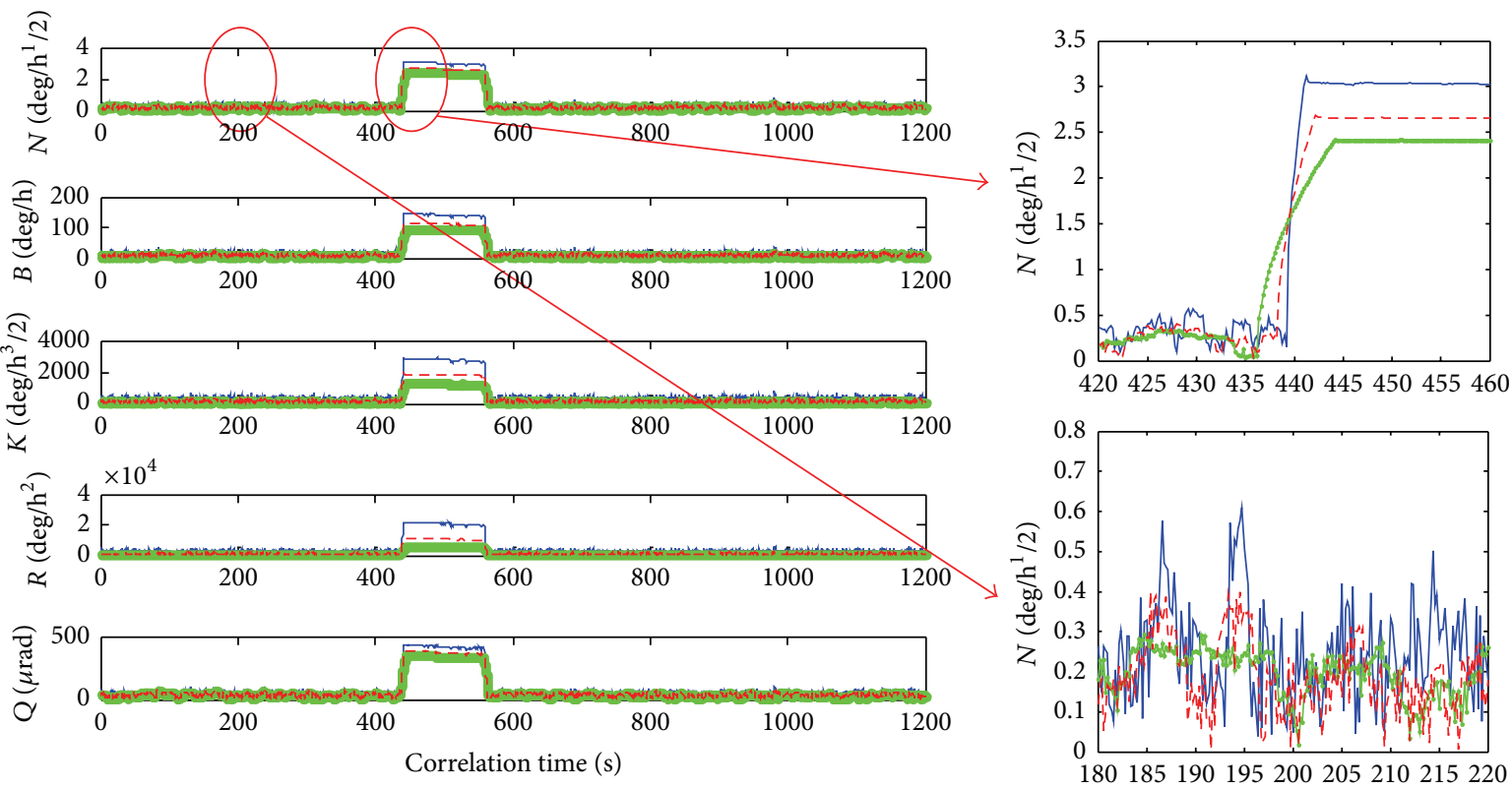

- SDAVAR

$\rightarrow$ LDAVAR

- - - Proposed algorithm

FIGURE 7: Curves of Allan various coefficients.

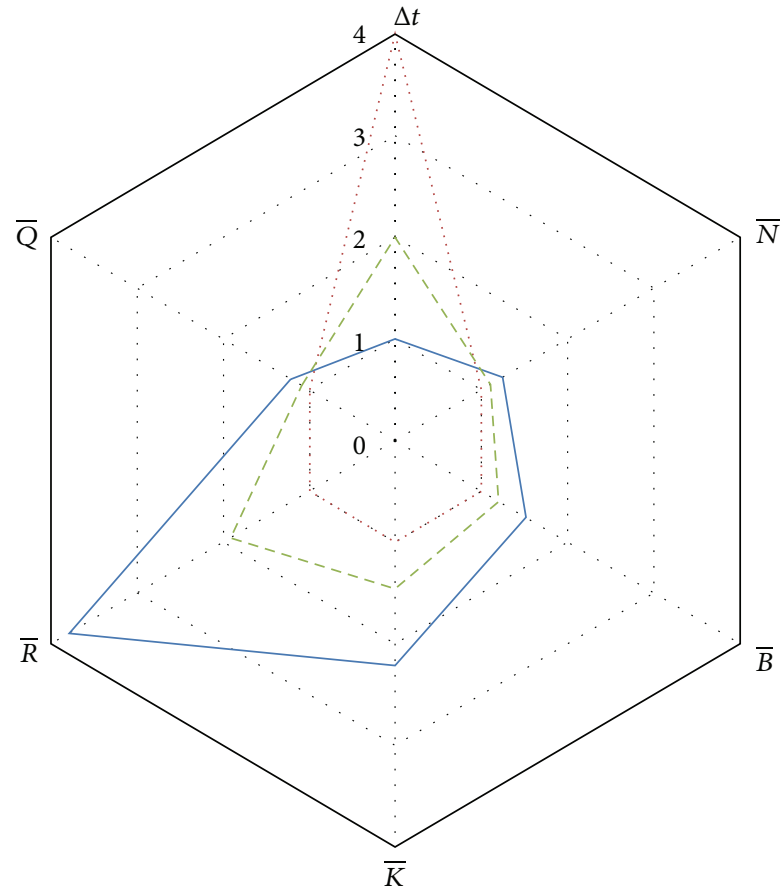

- SDAVAR

..... LDAVAR

- . - Proposed algorithm

FIGURE 8: Radar chart of algorithm performance evaluation.

algorithm provides an effective tool to evaluate the influences on the nonstationary FOG performances.

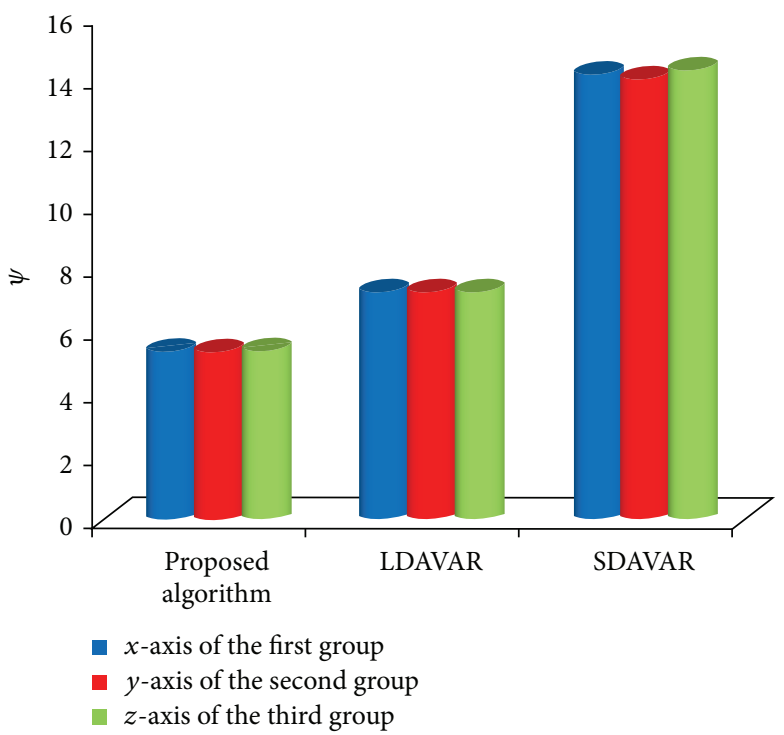

FIGURE 9: Comparison of performance evaluation indicators.

\section{Conflict of Interests}

The authors declare that there is no conflict of interests regarding the publication of this paper.

\section{Acknowledgments}

This work is supported by the National Natural Science Foundation of China (nos. 61374115, 61328301, 61104188, 
61273057, and 61174197) and by Foundation of Jiangsu Key Laboratory "Internet of Things and Control Technologies" and partly supported by the project funded by the Priority Academic Program Development of Jiangsu Higher Education Institutions.

\section{References}

[1] J. Z. Lai, P. Lv, J. Y. Liu, and B. Jiang, "Noncommutativity error analysis of strapdown inertial navigation system under the vibration in UAVs," International Journal of Advanced Robotic Systems, vol. 9, no. 136, pp. 1-8, 2012.

[2] R. Song, X. Chen, C. Shen, and H. Zhang, "Modeling FOG drift using back-propagation neural network optimized by artificial fish swarm algorithm," Journal of Sensors, vol. 2014, Article ID 273043, 6 pages, 2014.

[3] S. L. Han and J. L. Wang, "Quantization and colored noises error modeling for inertial sensors for GPS/INS integration," IEEE Sensors Journal, vol. 11, no. 6, pp. 1493-1503, 2011.

[4] V. V. Chikovani, "Performance parameters comparison of ring laser, coriolis vibratory and fiber-optic gyros based on Allan variance analysis," in Proceedings of the IEEE 2nd International Conference on Actual Problems of Unmanned Air Vehicles Developments (APUAVD 2013), pp. 153-156, October 2013.

[5] P. Lv, J. Liu, J. Lai, and G. Qin, "Research on the performance evaluation methods of fiber optical gyro stochastic errors," Chinese Journal of Scientific Instrument, vol. 35, no. 2, pp. 412418, 2014.

[6] L. Galleani and P. Tavella, "The dynamic allan variance," IEEE Transactions on Ultrasonics, Ferroelectrics, and Frequency Control, vol. 56, no. 3, pp. 450-464, 2009.

[7] L. Galleani, "The dynamic Allan variance II: a fast computational algorithm," IEEE Transactions on Ultrasonics, Ferroelectrics, and Frequency Control, vol. 57, no. 1, pp. 182-188, 2010.

[8] I. Sesia, L. Galleani, and P. Tavella, "Application of the dynamic allan variance for the characterization of space clock behavior," IEEE Transactions on Aerospace and Electronic Systems, vol. 47, no. 2, pp. 884-895, 2011.

[9] X. Li and N. Zhang, "Analysis of dynamic characteristics of a fiber-optic gyroscope based on dynamic Allan variance," Journal of Harbin Engineering University, vol. 32, no. 2, pp. 183187, 2011.

[10] M. A. Olivares-Mendez, I. F. Mondragón, and P. Campoy, "Autonomous landing of an unmanned aerial vehicle using image-based fuzzy control," Research, Education and Development of Unmanned Aerial Systems, vol. 2, no. 1, pp. 79-86, 2013.

[11] H.-Y. Chung, C.-C. Hou, and S.-C. Liu, "Automatic navigation of a wheeled mobile robot using particle swarm optimization and fuzzy control," in Proceedings of the IEEE 22nd International Symposium on Industrial Electronics (ISIE '13), pp. 1-6, IEEE, May 2013.

[12] F. Boufera, F. Debbat, F. Mondada, and M. F. Khelfi, "Fuzzy control system for autonomous navigation of thymio II mobile robots," Journal of Emerging Technologies in Web Intelligence, vol. 6, no. 1, pp. 101-105, 2014.

[13] J. Li, F. Gao, G. Wang, W. Gao, W. Zhu, and M. Wang, "Analysis of dynamic Allan variance for fiber optic gyro under vibration and variable temperature conditions," Chinese Journal of Lasers, vol. 40, no. 9, pp. 184-190, 2013.

[14] L. X. Zhu, Y. Lu, J. N. Xu, and X. H. Cheng, "Adaptive fuzzy-PID hybrid control method of gyro stabilized platform," Journal of Chinese Inertial Technology, vol. 22, no. 3, pp. 317-321, 2014.
[15] H. J. Li, Z. Q. Li, J. X. Peng, and L. H. Zhang, "Research on interactive visualization clustering method based on the radar chart," Applied Mechanics and Materials, vol. 241, pp. 1633-1639, 2013. 

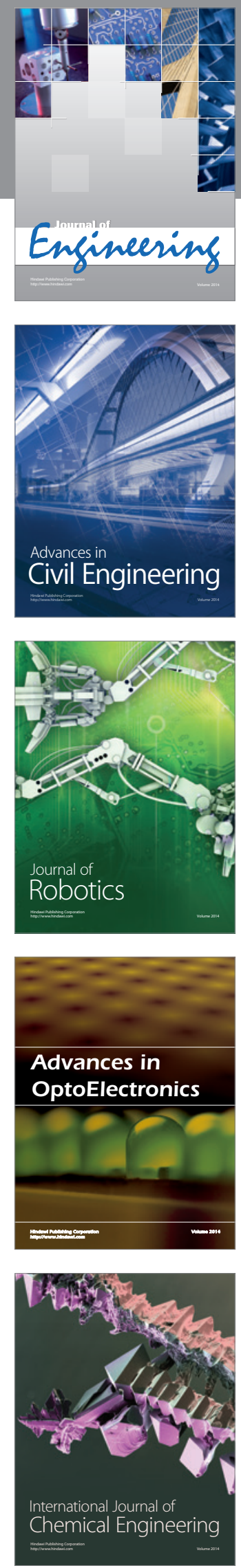

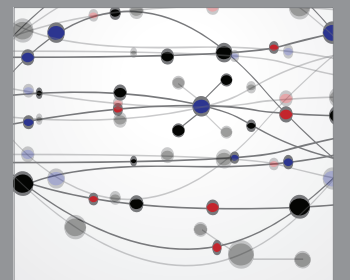

The Scientific World Journal
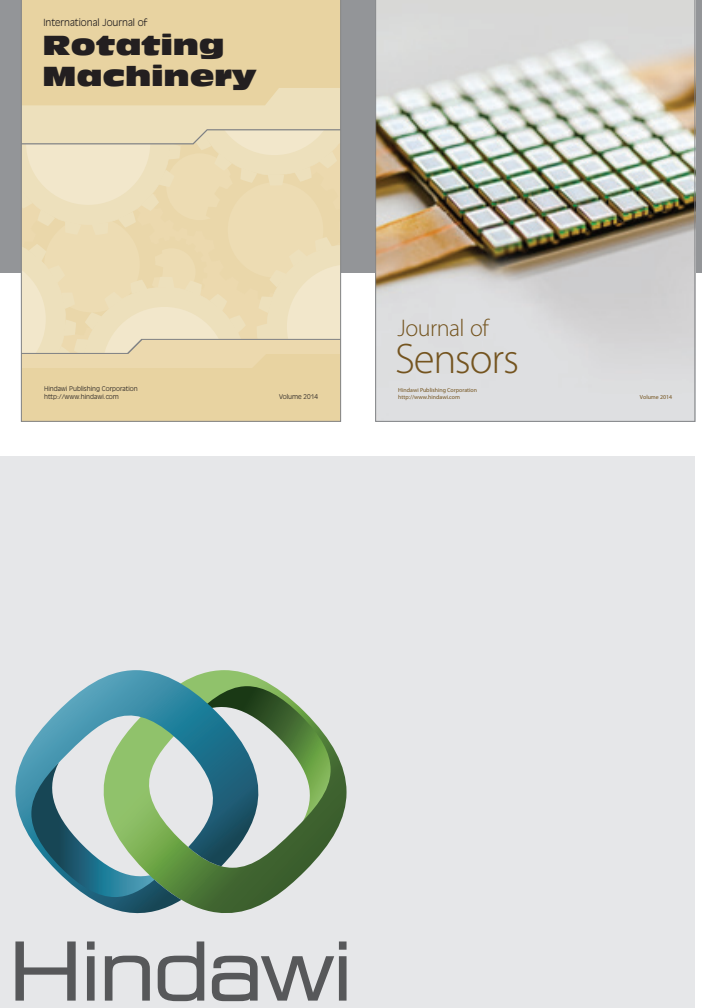

Submit your manuscripts at http://www.hindawi.com
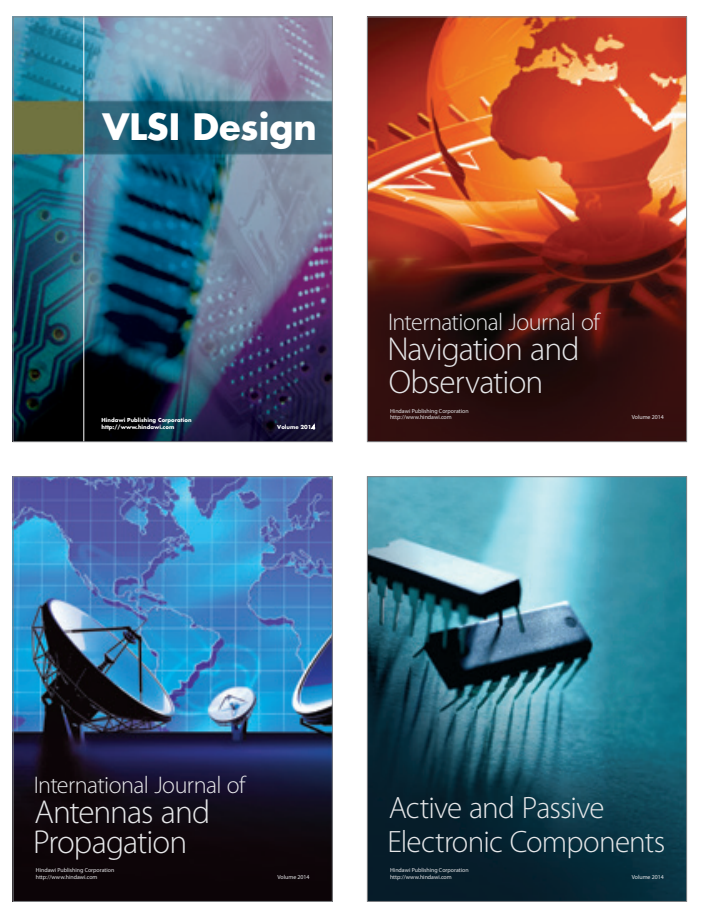
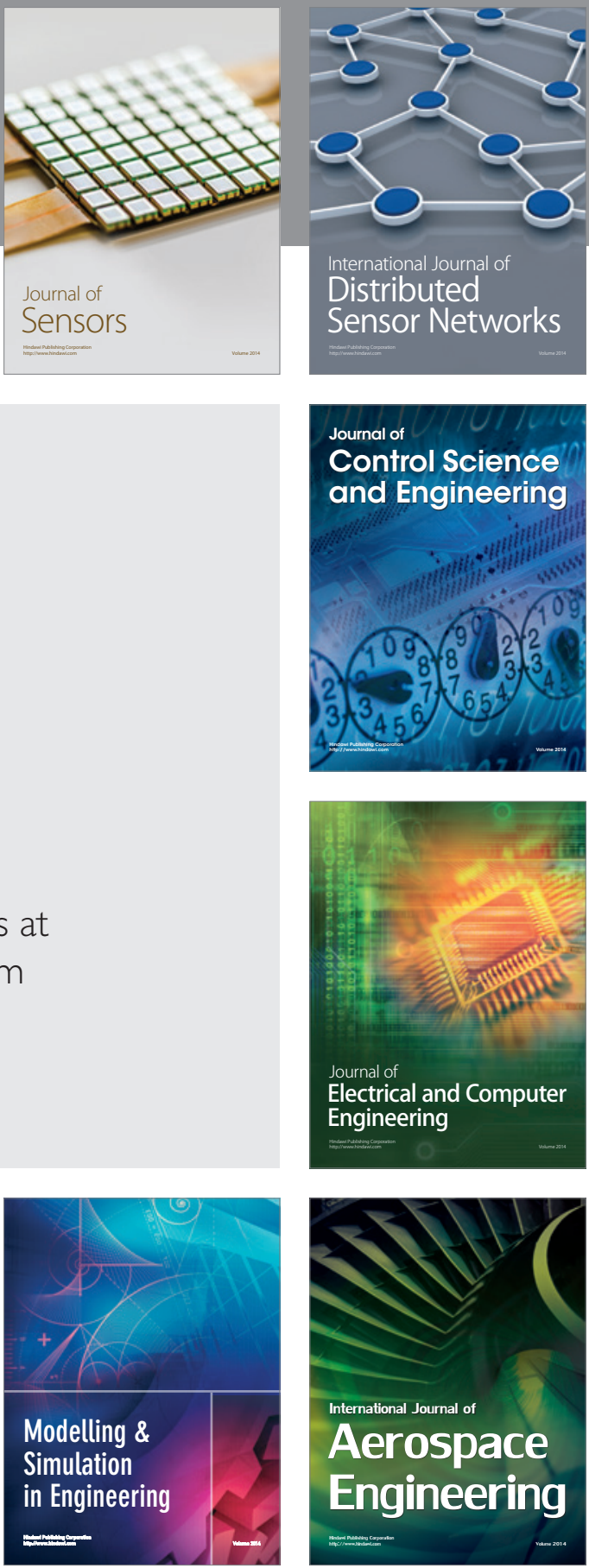

Journal of

Control Science

and Engineering
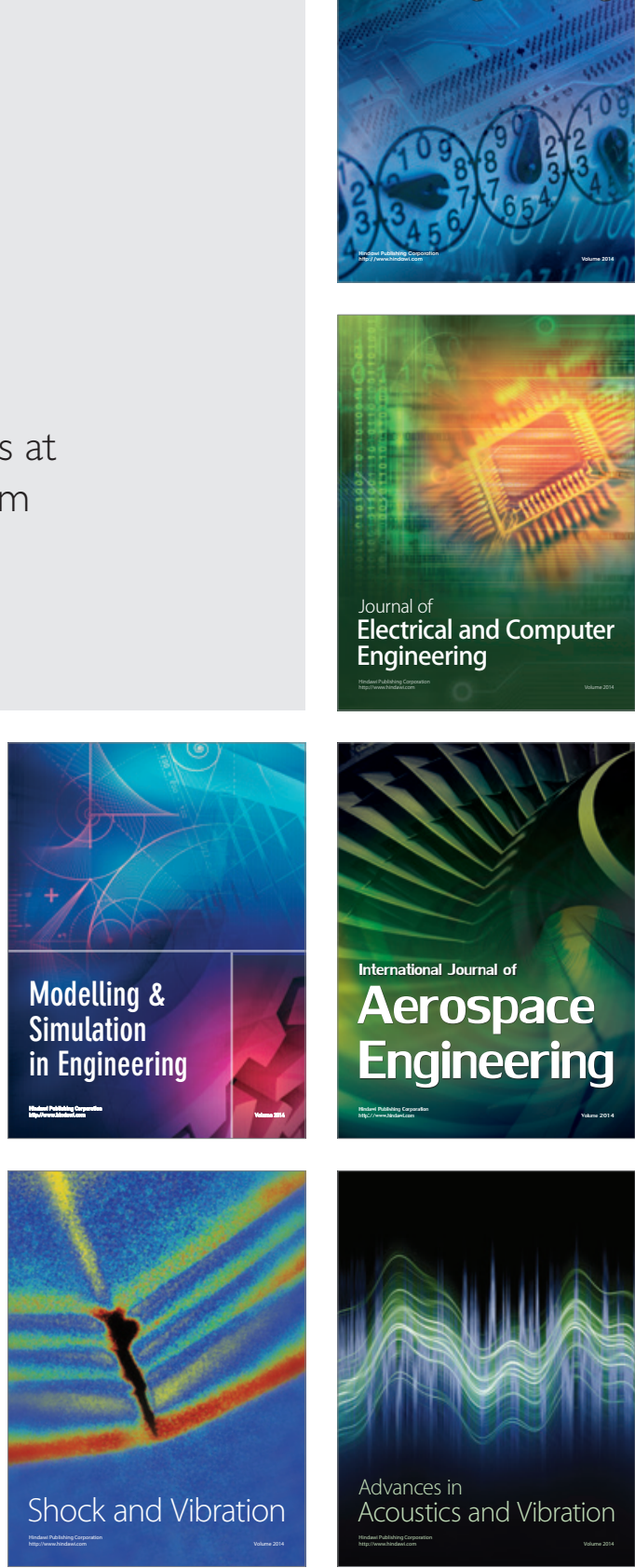